Trauma Berufskrankh 2014 · 16[Suppl 3]:231-233 DOI 10.1007/s10039-014-2054-x

Online publiziert: 19. April 2014

(c) Springer-Verlag Berlin Heidelberg 2014
W. Miller

Leinfelden-Echterdingen

\section{Erwartungen niedergelassener D-Ärzte an das stationäre Heilverfahren}

\author{
Neuausrichtung des stationären \\ Heilverfahrens der DGUV \\ (Deutsche Gesetzliche Unfallversicherung)
}

\section{Hintergrund}

Die Neuordnung des stationären Heilverfahrens betrifft auch die niedergelassenen D-Ärzte (Durchgangsärzte). Nach der am 31.12.2013 endenden Übergangsfrist sind Unfallverletzte, die der stationären Heilbehandlung bedürfen, nach den neuen Regeln dem Krankenhaus zuzuleiten. Einerseits entfällt für einige leichtere Verletzungen die Vorstellungspflicht im VAVHaus (VAV: Verletzungsartenverfahren), andererseits müssen besonders schwere Verletzungen jetzt primär in den neu definierten SAV-Kliniken (SAV: Schwerstverletzungsartenverfahren) versorgt werden. Dies ist sowohl im Ballungsraum als auch auf dem Land realisierbar, Voraussetzung dafür ist jedoch die enge Kommunikation zwischen BG (Berufsgenossenschaft), Krankenhaus und D-Arzt.

\section{Erwartungen der D-Ärzte}

\section{Allgemein}

Der D-Arzt erwartet vom neuen stationären Heilverfahren, dass es reibungslos funktioniert. In Gesprächen im Vorfeld signalisierten die niedergelassenen D-Arzt-Kollegen eine grundsätzliche $\mathrm{Zu}$ stimmung zum bisherigen Verfahren und zur aktuellen Weiterentwicklung - die Pa- tienten sollen in der optimalen Einrichtung versorgt werden.

Regeln zur Zuordnung galten auch bisher, diese werden jetzt modifiziert und fortgeschrieben. Allerdings sollten sie auch begründete Ausnahmen zulassen.

Es gibt kein Stadt-Land-Gefälle bezüglich der Wünsche. In der Fläche leben zwar weniger Menschen, und die Wege sind weiter, dafür aber sind die Straßen leerer. Der Weg ins nächste SAV-Haus dauert im Allgäu insgesamt nicht länger als im Ballungsraum Stuttgart. Schneeverwehungen gibt es dort nur an wenigen Tagen im Jahr, den Stau im Berufsverkehr in Stuttgart dagegen täglich. Der Weg ins nächste qualifizierte Krankenhaus stellt keine Barriere dar, weder für die Akutvorstellung noch für erforderliche Heilverfahrenskontrollen.

\section{Regelungswerk}

Die bisweilen geäußerte Befürchtung, eine weitere Differenzierung der Klinikkategorien würde die Versorgung in der Fläche gefährden, wird gerade von Kollegen im ländlichen Raum nicht geteilt. Bei näherer Betrachtung verfügt der südwestdeutsche Raum über ein historisch gewachsenes Krankenhausangebot, das nahezu überall auch spezialisierte Kliniken im Umkreis von $50 \mathrm{~km}$ erreichbar macht.
Die Erwartungen der D-Ärzte:

- eine gute Versorgung der Patienten,

- ordentliche Arbeitsbedingungen,

- sinnvolle Arbeitsabläufe,

- Wertschätzung der täglichen Arbeit durch die Patienten und auch die BG

decken sich in vielen Bereichen mit denen der Kliniken, der Patienten und auch der Berufsgenossenschaften.

\section{Flexible Heilverfahrenssteuerung}

Die Berufsgenossenschaft hat einen klaren Auftrag, Prävention und Rehabilitation aktiv mit „allen geeigneten Mitteln“ zu betreiben. Im Gegensatz dazu haben die gesetzlichen Krankenkassen lediglich die Aufgabe, Leistungen unter Beachtung des Wirtschaftlichkeitsgebots „zur Verfügung zu stellen“. Dieser Unterschied wird gelebt und auch wahrgenommen.

Die geringere Zahl der Verletzten in der Durchgangsarztpraxis bedarf der stationären Behandlung, die weitaus meisten können vom D-Arzt ambulant untersucht und definitiv versorgt werden. Für komplexe Fragestellungen allerdings werden spezialisierte Einrichtungen benötigt. Flexible Lösungen sind für Grenzfälle erforderlich, die nach der Diagnose obligat in einer VAV- oder SAV-Klinik behandelt werden müssten, aber ebenso gut 
ambulant beim D-Arzt oder im stationären DAV (D-Arzt-Verfahren) versorgt werden können. Entscheidend soll in diesem Fall eine rasche und gute Versorgung im Sinne des Verletzten sein. Daher wünschen sich die niedergelassenen D-Ärzte ein lernendes System mit der Perspektive, Regelungsinhalte den Bedürfnissen anzupassen, auch in kürzeren Zeitabständen von wenigen Jahren. Die Erfahrung zeigt: Sinnvolle Regeln bleiben, unsinnige verschwinden wieder.

\section{Kommunikation}

Verbesserungspotenzial besteht auch im Entlassungsmanagement der Kliniken und in der Heilverfahrenssteuerung durch die BG. Neben einer zeitnahen Informationsweitergabe sollen v. a. komplexe Fälle kurz direkt mit dem Nachbehandler besprochen werden. Das spart für alle Beteiligten mehr Zeit als es kostet. Eine erforderlich klinische Kontrolluntersuchung soll an dem Ort stattfinden, an dem auch die definitive Versorgung stattfand bzw. geplant ist. Zudem muss der D-Arzt hierüber zeitnah informiert werden. Sehr ungünstig ist es, wenn dem behandelnden D-Arzt ohne Information seitens der BG das Heilverfahren entzogen wird. In diesen oft ohnehin schwierigen Fällen wird der Patient zusätzlich verunsichert, und die Compliance wird gefährdet.

Neben den das stationäre Heilverfahren betreffenden Wünschen wird von den D-Ärzten, auch von denjenigen, die am Krankenhaus tätig sind, noch immer teilweise ein willkürlich erlebter Umgang mancher Berufsgenossenschaften und Unfallkassen mit Berichten und Leistungsabrechnungen beklagt. Es darf nicht sein, dass medizinisch sinnvolle Leistungen nicht erbracht werden und/oder die Dokumentation zurückgestellt wird, weil der D-Arzt damit rechnen muss, dass sie nicht vergütet werden. Auch hier wünschen sich die Kollegen v. a. mehr Dialog im Einzelfall.

\section{Verbesserung der Schnittstelle ambulant-stationär}

Indirekt zum stationären Heilverfahren, unmittelbar zur Schnittstelle ambulantstationär, gehören die Bitte und das An-

Trauma Berufskrankh 2014 · 16[Suppl 3]:231-233 DOI 10.1007/s10039-014-2054-x

(c) Springer-Verlag Berlin Heidelberg 2014

\section{W. Miller \\ Erwartungen niedergelassener D-Ärzte an das stationäre Heilverfahren. Neuausrichtung des stationären Heilverfahrens der DGUV (Deutsche Gesetzliche Unfallversicherung)}

\section{Zusammenfassung}

Hintergrund. Der niedergelassene Durchgangsarzt (D-Arzt) ist für viele Verletzte die erste Anlaufstelle. Er erwartet vom Heilverfahren die bestmögliche Rehabilitation der Verletzten.

Heilverfahren. Bereits vor Einführung des neuen stationären Heilverfahrens gab es unterschiedliche, von der Art und der Schwere der Verletzung abhängige Versorgungspfade. Im neuen stationären Heilverfahren werden diese Vorgaben angepasst und um die primäre Vorstellungspflicht im SAV-Haus (SAV: Schwerstverletzungsartenverfahren) für besonders Verletzte erweitert. Ausdrücklich begrüßt wird die Möglichkeit, weniger schwer Verletzte, die auch in kleineren Kliniken optimal versorgt werden können, dort definitiv zu behandeln. Die Vorstellung bestimmter Schwerverletzter in spezialisierten Kliniken war bereits früher praktizierte Reali- tät. Für eine optimale Versorgung sind auch längere Transportwege akzeptabel.

Erwartungen der niedergelassenen D-Ärzte. Hauptwunsch der D-Ärzte ist eine bessere Kommunikation zwischen Klinik, Berufsgenossenschaft und Durchgangsarzt. Trotz elektronischer Datenübermittlung ist der persönliche Austausch, zeitnah telefonisch und durch regelmäßige Treffen, nicht zu ersetzen. Wünschenswert ist außerdem eine Hospitation bzw. Weiterbildung der jüngeren Klinikärzte beim niedergelassenen D-Arzt, um diese Versorgungsstufe kennenzulernen und besser zu verstehen.

\section{Schlüsselwörter}

Arbeitsunfall · Unfallversicherung .

Stationäres Heilverfahren .

Berufsgenossenschaft · Kommunikation

\section{Expectancies of the "Durchgangsarzt". New procedure for inpatient treatment after injuries}

\section{Abstract}

Background. The German "Durchgangsarzt/ D-Arzt" (i.e. a trauma physician with his own office) is the first point of contact for many injured persons. His goal is to achieve the best possible treatment for his patients.

Treatment. The treatment paths depend on the severity of injury. According to the new SAV ("Schwerstverletzungsartenverfahren", i.e. a procedure for inpatient treatment after very severe injuries) these patients must be presented to a certified SAV hospital. Patients with less severe injuries are allowed to be treated in smaller local trauma hospitals, if the treatment complies with the conditions. The presentation of severely injured patients to specialized hospitals was previously practiced, but without these strict rules. For optimal treatment, longer transport distances are also acceptable.
Expectations of a D-Arzt. The main wish of the D-Arzt is improved communication between the hospital, the occupational insurance association, and the local trauma physician. Despite the possibilities of electronic data transfer, personal contact (e.g., by telephone or regular meetings) cannot be replaced. To better understand the working conditions and the problems of a D-Arzt, less experienced hospital physicians are invited for an internship in a D-Arzt office.

\section{Keywords}

Accidents, occupational - Accident insurance Inpatient treatment - Occupational insurance association · Communication gebot an die leitenden Krankenhausärzte, ihre nachgeordneten Ärzte zur Hospitation oder auch zum Ableisten von Weiterbildungsabschnitten zu niedergelassenen D-Ärzten zu schicken. Hier lassen sich einerseits Weiterbildungsinhalte wie Röntgendiagnostik, Sonographie, Arth- roskopie, aber auch das Management der Arbeitsunfähigkeit mit Lohnfortzahlung, Verletztengeld und Arbeits- und Belastungserprobung sowie die Handhabung von Bagatellverletzungen, die nach Wochen noch Beschwerden und Kosten verursachen, ideal erlernen. Viele D-Ärzte 
würden diese Kollegen gerne regulär als Weiterbildungsassistenten beschäftigen, wenn sie kämen.

\section{Gemeinsames Ziel}

Die optimale Versorgung der Verletzten ist das gemeinsame Ziel aller Beteiligten.

Der Arztberuf als freier Beruf ist primär dem Wohl des Patienten verpflichtet. Nach der Berufsordnung gehören dazu die Gesundheitsvorsorge ebenso wie das Heilen von Krankheiten. Der ideale Partner hierfür ist die BG. Deren Auftrag sind die optimale Prävention und Rehabilitation der Beschäftigten bzw. Verletzten. Das berufsgenossenschaftliche Heilverfahren bewährte sich über Jahrzehnte, und das Wort Arbeitsunfall ist nach wie vor ein Türöffner. Das ist auch das Verdienst der konsequenten Arbeit der Berufsgenossenschaften, die allen Gesundheitsreformen zum Trotz bis heute den verletzten Menschen und nicht die Sozialpolitik in den Mittelpunkt ihrer Arbeit stellen. Dafür danken die Durchgangsärzte nicht nur den Kliniken mit Pflegedienst, Therapeuten, Ärzten und Management, sondern auch den Mitarbeitern der Berufsgenossenschaften für ihre tägliche Arbeit in der Prävention für die Beschäftigten und in der Rehabilitation unserer Verletzten.

\section{Korrespondenzadresse}

Dr.W. Miller
Martin-Luther-Straße 7,
70771 Leinfelden-
Echterdingen
praxis@wolfgang-miller.de

\section{Einhaltung ethischer Richtlinien}

Interessenkonflikt. W. Miller gibt an, dass kein Interessenkonflikt besteht.

Dieser Beitrag beinhaltet keine Studien an Menschen oder Tieren.

The supplement containing this article is not sponsored by industry. 